\title{
Nuclear Broadening Effects on Hard Prompt Photons at Relativistic Energies
}

\author{
A. Dumitru ${ }^{a}$, L. Frankfurt ${ }^{b, c}$, L. Gerland ${ }^{b}$, H. Stöcker ${ }^{b}$, M. Strikman ${ }^{d}$ \\ a Physics Department, Columbia University, 538 West 120th Street, New York, NY10027, USA \\ b Institut für Theoretische Physik der J.W.Goethe-Universität \\ Robert-Mayer-Str. 8-10, D-60054 Frankfurt a.M., Germany \\ c Tel Aviv University, Tel Aviv, Israel \\ d Department of Physics, Pennsylvania State University, University Park, PA 16802, USA
}

(November 21, 2018)

\begin{abstract}
We calculate prompt photon production in high-energy nuclear collisions. We focus on the broadening of the intrinsic transverse momenta of the partons in the initial state from nuclear effects, and their influence on the prompt photon $p_{t}$ distribution. Comparing to WA98 data from $\mathrm{Pb}+\mathrm{Pb}$ collisions at $\sqrt{s}=17.4 A \mathrm{GeV}$ we find evidence for the presence of nuclear broadening at high $p_{t}$ in this hard process. Below $p_{t} \sim 2.7 \mathrm{GeV}$ the photon distribution is due to small momentum transfer processes. At RHIC energy, $\sqrt{s}=200 A \mathrm{GeV}$, the effect of intrinsic transverse momentum on the spectrum of prompt photons is less prominent. The region $p_{t}=3-4 \mathrm{GeV}$ would be the most promising for studying the nuclear broadening effects at that energy. Below $p_{t}=2-3 \mathrm{GeV}$ the contribution from large momentum transfers flattens out, and we expect that region to be dominated by soft contributions.
\end{abstract}

\section{INTRODUCTION}

The WA98 collaboration recently reported data on direct photon production in lead on lead collisions at CERN SPS energies $(\sqrt{s}=17.4 A \mathrm{GeV})$ at $p_{t} \sim 1.5-4 \mathrm{GeV}$ [1]. They made the interesting observation that in central $\mathrm{Pb}+\mathrm{Pb}$ collisions the multiplicity of direct photons per nucleon-nucleon collision in that range of $p_{t}$ is enhanced relative to proton- 
proton collisions at similar energy. (For a review of data and calculations of single photon production in proton and pion induced scattering off various targets see [2].) It is the purpose of this paper to analyze to what extent, and in which range of $p_{t}$, that data can be understood within perturbative QCD (pQCD), after introducing an intrinsic transverse momentum of the partons. That transverse momentum is due to the partons being confined in the initial-state nucleons, gluon bremsstrahlung, as well as from multiple soft scattering of the nucleons prior to the hard scattering. It can be identified in the nuclear dependence of the intrinsic transverse momentum of the partons predicted in [3] and observed recently for dimuon production in $p+A$ collisions $[4$.

Thus, the purpose of this paper is to analyse the direct photon data of ref. [1] to probe nuclear broadening effects on the transverse momentum distribution of direct photons. Those effects are expected to increase the prompt photon cross section strongly, because a part of the $p_{t}$ of the photon can be "supplied" by the incoming partons rather than in the elementary semi-hard scattering itself [5].

Even so, we explicitly restrict the computation to include only the contribution from parton-parton scattering amplitudes at a minimum momentum transfer of $\geq 1 \mathrm{GeV}$. Thus, comparing the calculated $p_{t}$ distribution of photons to the data, one can determine the $p_{t}$ scale below which production of single photons is dominated by reactions at small momentum transfer, $<1 \mathrm{GeV}$. That is probably the region where a pQCD description looks suspicious. Note that without imposing a cut on the minimum momentum transfer in elementary partonparton scatterings the perturbative contribution becomes very large at low $p_{t}$, precisely because of the fact that $p_{t} \sim 1-2 \mathrm{GeV}$ photons can be produced with almost no momentum transfer, the $p_{t}$ being supplied by the intrinsic $k_{t}$ of the partons. Therefore, care must be taken when comparing perturbative computations accounting for the violation of the DGLAP approximation due to parton intrinsic transverse momentum to data. This primary transverse momentum is to large extent due to the gluon bremsstrahlung. With increasing energy, primary transverse momentum plays a less important role because a lot of gluons are radiated "long before" the hard collision [6]. Thus the distribution of partons near the point 
of the hard collision will be determined mostly by gluon bremstrahlung, which is universal - independent of the target.

We shall also make predictions for $\mathrm{Au}+\mathrm{Au}$ collisions at nominal BNL-RHIC energy, $\sqrt{s}=$ $200 A \mathrm{GeV}$. Prompt photon spectra at RHIC will be measured in the future by the PHENIX collaboration. We shall discuss the importance of intrinsic transverse momentum at that energy as well, and identify the $p_{t}$ where the photon distribution from pQCD interactions with momentum transfer $\geq 1 \mathrm{GeV}$ flattens out.

The significant effects from nuclear broadening of the transverse parton distribution on photon production in $A+A$ collisions are in general agreement with the description of the yield of electrically neutral pions in [7]. It proved essential for the understanding of $\pi^{0}$ production in $\mathrm{Pb}+\mathrm{Pb}$ at $\mathrm{SPS}$ energy 8$]\left(\sqrt{s}=17.4 A \mathrm{GeV} ; p_{t}=1-4 \mathrm{GeV}\right)$ to account for the $p_{t}$-broadening. Also, measurements of both the prompt photon and pion production cross section in future $p+A$ experiments at BNL-RHIC can determine the scaling of the nuclear broadening with $A$ [7,g]; that could provide additional information to that obtained from the Drell-Yan process and $J / \Psi, \Upsilon$ production.

\section{PROMPT PHOTON $P_{T}$ DISTRIBUTION IN $P+P$}

We consider the contributions from Compton-like scattering $(g+\stackrel{(-)}{q} \rightarrow \gamma+\stackrel{(-)}{q})$, annihilation $(q+\bar{q} \rightarrow \gamma+g)$, plus collinear bremsstrahlung off a (anti-)quark produced at midrapidity. Assuming the applicability of the QCD factorization theorem, the corresponding expressions for the Compton and annihilation subprocesses in $p+p \rightarrow \gamma+X$ are [5]

$$
E \frac{\mathrm{d} \sigma_{\gamma}}{\mathrm{d}^{3} p}=\sum \int \mathrm{d} x_{a} \mathrm{~d} x_{b} \mathrm{~d}^{2} k_{t a} \mathrm{~d}^{2} k_{t b} f\left(k_{t a}\right) f\left(k_{t b}\right) G_{a / A}\left(x_{a}, Q^{2}\right) G_{b / B}\left(x_{b}, Q^{2}\right) \frac{\hat{s}}{\pi} \frac{\mathrm{d} \sigma}{\mathrm{d} \hat{t}} \delta(\hat{s}+\hat{t}+\hat{u}) \Theta_{0}
$$

$\hat{s}, \hat{t}, \hat{u}$ denote the Mandelstam variables for the $a+b \rightarrow \gamma+c$ elementary process. The sum extends over all possible partons in the initial state, i.e. gluons and $u, d, s$ (anti-)quarks, and over any final state interactions. $\mathrm{d} \sigma / \mathrm{d} \hat{t}$ denotes the elementary hard-scattering cross 
section for the corresponding process, averaged over all possible spin and color orientations in the initial state, and summed over those in the final state. Explicit expressions can be found e.g. in [5].

The $G_{a / A}\left(x_{a}, Q^{2}\right)$ are the twist-2 infrared dominated matrix elements, for which we employ the CTEQ4L parametrization [10]. (We consider only the processes with unpolarized particles, so twist- 4 would be the next to contribute.) At rather small- $x$, nuclear shadowing of the parton distribution functions may result in considerable suppression of the gluon and sea-quark distributions at moderate values of $Q^{2}$ [11]. This may then in turn affect the $p_{t}$ distribution of prompt photons at collider energies (RHIC, LHC) in the $p_{t}$-range of a few $\mathrm{GeV}$ [12,13]. On the other hand, at CERN-SPS energy, $\sqrt{s} \simeq 17-20 A \mathrm{GeV}$, and for $p_{t} \sim 1 \mathrm{GeV}$, we rather deal with the parton distributions at $x \sim 0.1$, which is in the domain of antishadowing effects. The antishadowing effect is rather small, however: EKS [14] and FGS [15] estimates predict about 10\% - 15\% enhancement. As we shall see below, this appears to be well within the uncertainties of the value of the intrinsic transverse momentum, and thus will not be considered here in more detail.

The functions $f\left(k_{t}\right)$ parametrize the transverse momentum of the partons in the initial state. For simplicity, we assume a Gaussian distribution,

$$
f\left(k_{t}\right)=\frac{1}{\pi\left\langle k_{t}^{2}\right\rangle} e^{-k_{t}^{2} /\left\langle k_{t}^{2}\right\rangle}
$$

The distribution (2) contains one tunable parameter, namely the average intrinsic transverse momentum of the partons in the initial state, $\left\langle k_{t}^{2}\right\rangle$. We shall present results for various values of $\left\langle k_{t}^{2}\right\rangle$ below. The limit $\left\langle k_{t}^{2}\right\rangle \rightarrow 0$ recovers the usual collinear factorization.

The intrinsic $k_{t}$ for plane waves bound in a nucleon can be estimated by applying the uncertainty principle:

$$
\sqrt{k_{t}^{2}} \approx \frac{\pi}{2 r_{N}} \approx 0.37 \mathrm{GeV}
$$

Here, $r_{N} \approx 0.85 \mathrm{fm}$ is the radius of a nucleon. Initial state gluon bremsstrahlung can give rise to larger intrinsic $k_{t}$ on the order of $1 \mathrm{GeV}$. 
For the factorization scale we assume $Q^{2}=\left(2 p_{t}\right)^{2}$, and as we restrict ourselves to the leading logarithmic approximation, we assume that the renormalization scale is the same. We employ the one-loop expression for the running coupling constant $\alpha_{s}\left(Q^{2}\right)$ for $N_{f}=4$, and $\Lambda_{\mathrm{QCD}}=236 \mathrm{MeV}$, as appropriate for CTEQ4L. Collinear divergencies arising from partonic processes with large intrinsic transverse momentum are cut off by

$$
\Theta_{0}=\Theta_{1} \Theta_{2} \Theta\left(Q_{c}^{2}-1 \mathrm{GeV}^{2}\right)
$$

where we define

$$
Q_{c}^{2}=\frac{2 \hat{s} \hat{t} \hat{u}}{\hat{s}^{2}+\hat{t}^{2}+\hat{u}^{2}}
$$

In this way, only subprocesses with momentum transfer $\geq 1 \mathrm{GeV}$ are taken into account, for which one can hope that factorization is a reasonable assumption. The functions $\Theta_{1}$ and $\Theta_{2}$ ensure that parton $a$ is moving to the right, while parton $b$ is moving to the left,

$$
\Theta_{1}=\Theta\left(k_{z a}\right) \quad, \quad \Theta_{2}=\Theta\left(-k_{z b}\right)
$$

Using on-shell kinematics, the four-momenta of the incoming partons are given by

$$
\begin{aligned}
\left(E_{a}, \vec{k}_{t a}, k_{z a}\right) & =\left(\frac{1}{2} x_{a} \sqrt{s}+\frac{k_{t a}^{2}}{2 x_{a} \sqrt{s}}, \vec{k}_{t a}, \frac{1}{2} x_{a} \sqrt{s}-\frac{k_{t a}^{2}}{2 x_{a} \sqrt{s}}\right) \\
\left(E_{b}, \vec{k}_{t b}, k_{z b}\right) & =\left(\frac{1}{2} x_{b} \sqrt{s}+\frac{k_{t b}^{2}}{2 x_{b} \sqrt{s}}, \vec{k}_{t b},-\frac{1}{2} x_{b} \sqrt{s}+\frac{k_{t b}^{2}}{2 x_{b} \sqrt{s}}\right) .
\end{aligned}
$$

After using the $\delta$-function in eq. (1) to solve for $x_{b}$, the remaining integrals can be performed numerically via Monte-Carlo techniques. Numerical convergence can be checked by comparing spectra obtained with varying sequences of random numbers and increasing the number of Monte-Carlo points.

Collinear bremsstrahlung is computed as follows. First, the cross-section for (anti-)quark production at $y_{c m}=0$ with transverse momentum $p_{t c}$ is computed in the standard fashion, as described above. This is then convoluted with the QED collinear fragmentation function $z_{c} D_{\gamma / q}\left(z_{c}\right)=\alpha\left[1+\left(1-z_{c}\right)^{2}\right] \log \left(p_{t}^{2} / \Lambda_{\mathrm{QCD}}^{2}\right) / 2 \pi$ into $\gamma+X$, where $z_{c}=p_{t} / p_{t c}$ 


$$
\begin{aligned}
E \frac{\mathrm{d} \sigma_{\gamma}}{\mathrm{d}^{3} p}= & \sum \int \mathrm{d} x_{a} \mathrm{~d} x_{b} \mathrm{~d} z_{c} \mathrm{~d}^{2} k_{t a} \mathrm{~d}^{2} k_{t b} \\
& f\left(k_{t a}\right) f\left(k_{t b}\right) G_{a / A}\left(x_{a}, Q^{2}\right) G_{b / B}\left(x_{b}, Q^{2}\right) D_{\gamma / c}\left(z_{c}\right) \frac{\hat{s}}{\pi z_{c}^{2}} \frac{\mathrm{d} \sigma}{\mathrm{d} \hat{t}} \delta(\hat{s}+\hat{t}+\hat{u}) \Theta_{0}
\end{aligned}
$$

Here, we employ $Q^{2}=\left(2 p_{t c}\right)^{2}$ for the factorization and renormalization scale of the hard process. For the bremsstrahlung contribution we do not sum explicitly over all hard subprocesses leading to the production of a (anti-)quark at midrapidity but rather employ the method of effective structure function,

$$
G_{a / A}\left(x_{a}, Q^{2}\right) G_{b / B}\left(x_{b}, Q^{2}\right)=\left(g\left(x_{b}, Q^{2}\right)+\frac{4}{9} \sum_{f} q_{f}\left(x_{b}, Q^{2}\right)\right) \sum_{f} e_{f}^{2} q_{f}\left(x_{a}, Q^{2}\right)
$$

where $e_{f}$ is the fractional electric charge of the splitting (anti-)quark. Further, $\mathrm{d} \sigma / \mathrm{d} \hat{t}=$ $\pi\left(\alpha_{s} / \hat{s}\right)^{2}\left(\hat{s}^{2}+\hat{u}^{2}\right) / \hat{t}^{2}$. The $\Theta$-function is defined as in (4, 6). Note that in these expressions $\hat{s}, \hat{t}, \hat{u}$ refer to the partonic subprocess, "before" splitting of the final-state (anti-)quark into $\gamma+X$

It is common practice to multiply the r.h.s. of (1) and (9) by a $K$-factor in order to account for NLO contributions. Full NLO computations have been performed [16] for the case without intrinsic $k_{t}$ of the partons and indicate $K \simeq 1.8-2.0$ for $p+p$ collisions at energies around $\sqrt{s}=20 \mathrm{GeV}$, and for photon transverse momenta in the range $2-4 \mathrm{GeV}$. Our major interest is in the relative yields of prompt photons in $p+p$ and in $A+A$ collisions, and in that sense the $K$-factor plays no essential role. In any case, we shall fix the value of $K$ from $p+p$ and then keep its value for $A+A$. 


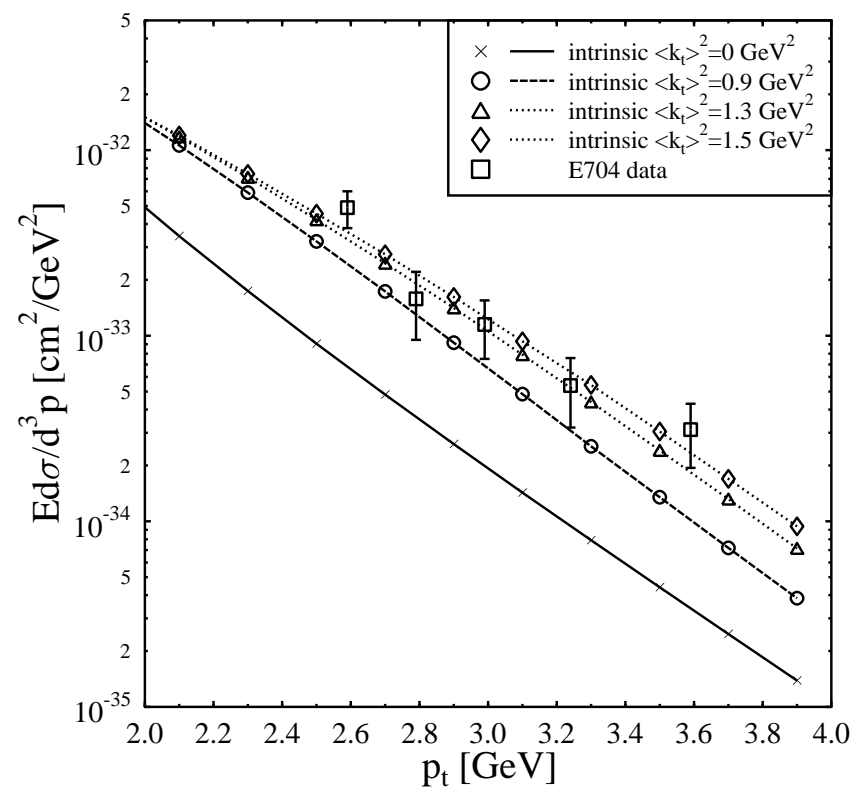

FIG. 1. The differential direct photon cross section vs. $p_{t}$ (at center-of-mass rapidity) for proton-proton collsions at $\sqrt{s}=19.4 \mathrm{GeV}$.

In Fig. 11 we compare our results for $p+p$ at $\sqrt{s}=19.4 \mathrm{GeV}$, for various intrinsic $\left\langle k_{t}^{2}\right\rangle$ and $K=2$, to E704 data [17]. Clearly, the calculation assuming pure DGLAP evolution, i.e. $\left\langle k_{t}^{2}\right\rangle=0$, falls short by almost an order of magnitude. The best fit is obtained using $\left\langle k_{t}^{2}\right\rangle=1.3-1.5 \mathrm{GeV}^{2}$. At first sight this appears somewhat larger than the "standard" $\left\langle k_{t}^{2}\right\rangle=0.9 \mathrm{GeV}^{2}$ from [5,18]. However, recall that we employ a larger $Q^{2}=4 p_{t}^{2}$ scale than [5, 18, who used $Q^{2}=p_{t}^{2} / 2$. In accord with the picture that the intrinsic transverse momentum is due mainly to gluon radiation in the initial state, a larger $Q^{2}$ scale therefore has to correspond to larger $\left\langle k_{t}^{2}\right\rangle$. This general expectation is confirmed by our results. (The value for $\left\langle k_{t}^{2}\right\rangle$ therefore only acquires physical meaning together with a definition of $Q^{2}$.)

The above calculation serves to fix the $K$-factor and the strength of the DGLAP violation on the $p+p$ level. From there we conclude that with $K=2, Q^{2}=4 p_{t}^{2}$, a value of $\left\langle k_{t}^{2}\right\rangle \simeq 1.3-1.5 \mathrm{GeV}^{2}$ is required to fit the data at that energy. 


\section{HARD PHOTON PRODUCTION IN NUCLEUS-NUCLEUS COLLISIONS}

In the previous section we discussed hard photon production in $p+p$ or $N+N$ collisions (we neglect the differences between protons and nucleons in our calculations). To extend our calculations to nucleus-nucleus collisions we have to substitute the parton distribution functions $G_{N}(x, Q)$ in eq. (11) by $G_{A}\left(x, Q, \boldsymbol{b}^{\prime}\right)$ for the projectile and $G_{B}\left(x, Q, \boldsymbol{b}-\boldsymbol{b}^{\prime}\right)$ for the target nucleus. Here, $G_{A}\left(x, Q, \boldsymbol{b}^{\prime}\right)=A \cdot T_{A}\left(\boldsymbol{b}^{\prime}\right) G_{N}(x, Q)$ and $G_{B}\left(x, Q, \boldsymbol{b}-\boldsymbol{b}^{\prime}\right)=B \cdot T_{B}(\boldsymbol{b}-$ $\left.\boldsymbol{b}^{\prime}\right) G_{N}(x, Q)$ [19], with $\boldsymbol{b}$ the nucleus-nucleus impact parameter, and $\boldsymbol{b}^{\prime}$ the distance from the center of the projectile. Note that $\boldsymbol{b}$ and $\boldsymbol{b}^{\prime}$ are two-dimensional vectors.

The nuclear thickness functions $T_{A, B}\left(\boldsymbol{b}^{\prime}\right)$ are defined as

$$
T_{A, B}\left(\boldsymbol{b}^{\prime}\right)=\int \mathrm{d} z \rho_{A, B}\left(\boldsymbol{b}^{\prime}, z\right) \text { with } \int \mathrm{d}^{2} \boldsymbol{b}^{\prime} \mathrm{d} z \rho\left(\boldsymbol{b}^{\prime}, z\right)=1
$$

$A(B)$ are the number of nucleons in the projectile (target). We account for the dependence on the nucleus nucleus impact parameter $b$ as due to the geometry of the collision only. If one integrates over all impact parameters to compare with minimum bias data, this yields

$$
\sigma_{\gamma}^{A B}=A B \cdot \sigma_{\gamma}^{N N}
$$

To compare to the experimental data by the WA98 collaboration we need to calculate the direct photon cross section for their centrality trigger. To take into account the dependence of the nuclear parton distribution functions on the geometry we employ the semiclassical approximation of the Glauber-model that was developed for hadron nucleus collisions in ref. [20] and extented in ref. [21] to nucleus nucleus collisions.

The inclusive spectrum of hard photons in $A+B$ collisions at the impact parameter $\boldsymbol{b}$ is

$$
E_{\gamma} \frac{\mathrm{d} N_{\gamma}^{A B}}{\mathrm{~d}^{3} \boldsymbol{p}_{\gamma}}(\boldsymbol{b})=A B \cdot T_{A B}(\boldsymbol{b}) E_{\gamma} \frac{\mathrm{d} \sigma_{\gamma}^{N N}}{\mathrm{~d}^{3} \boldsymbol{p}_{\gamma}} .
$$

$E_{\gamma}$ and $\boldsymbol{p}_{\gamma}$ are the energy and the momentum of the emitted photon, and $\sigma_{\gamma}$ is the inclusive cross section for direct photon production.

$T_{A B}$ is the nucleus-nucleus thickness function. For large nuclei with a slowly varying density distribution $T_{A B}$ is given by 


$$
T_{A B}(\boldsymbol{b})=\int \mathrm{d}^{2} \boldsymbol{b}^{\prime} T_{A}\left(\boldsymbol{b}^{\prime}\right) T_{B}\left(\boldsymbol{b}-\boldsymbol{b}^{\prime}\right)
$$

In a $A+B$ collision at impact parameter $\boldsymbol{b}$, the nucleus-nucleus thickness function allows only collisions of partons in overlapping slabs, i.e. of partons with the same coordinates in the plane transverse to the beam direction.

Finally, the differential number of direct photons in nucleus-nucleus collisions integrated over impact parameters $0<b<b_{m}$ is given by the ratio of the differential cross section to the inelastic nucleus-nucleus cross section, both being integrated over the region $\left[0, b_{m}\right]$ :

$$
E_{\gamma} \frac{\mathrm{d} N_{\gamma}^{A B}}{\mathrm{~d}^{3} \mathbf{p}_{\gamma}}\left(b_{m}\right)=\frac{E_{\gamma} \int_{0}^{b_{m}} \mathrm{~d}^{2} \boldsymbol{b} \frac{\mathrm{d} \sigma_{\gamma}^{A B}}{\mathrm{~d}^{3} \mathbf{p}_{\gamma}}(\boldsymbol{b})}{\sigma_{\text {inel }}^{A B}\left(b_{m}\right)}=\frac{E_{\gamma} \frac{\mathrm{d} \sigma_{p p}^{\gamma}}{\mathrm{d}^{3} \mathbf{p}_{\gamma}} \int_{0}^{b_{m}} \mathrm{~d}^{2} \boldsymbol{b} A B \cdot T_{A B}(\boldsymbol{b})}{\int_{0}^{b_{m}} \mathrm{~d}^{2} \boldsymbol{b}\left(1-\left(1-T_{A B}(\boldsymbol{b}) \sigma_{\text {in }}^{N N}\right)^{A B}\right)} .
$$

From this equation, we can also obtain the prompt photon multiplicity for $p+A$ scattering. To that end, the nucleus-nucleus thickness function $T_{A B}(\boldsymbol{b})$ has to be replaced by the nucleus thickness function $T_{A}(\boldsymbol{b})$, and $B=1$ throughout the equation. For $p+A$ we have $b_{m}=\infty$, corresponding to minimum bias scattering.

In our calculations we used the Woods-Saxon density distribution of ref. [22] and assumed $\sigma_{\text {in }}^{N N}=30 \mathrm{mb}$ at $\sqrt{s} \sim 20 \mathrm{~A} \mathrm{GeV}$. That yields a total inelastic cross section for $\mathrm{Pb}+\mathrm{Pb}$ of $\sigma_{t o t}^{P b P b}=7100 \mathrm{mb} . \sigma_{t o t}^{P b P b}$ is given by the denominator of eq. (15) with $b_{m}=\infty$. For large values of $A B$ one can approximate the denominator by

$$
\int \mathrm{d}^{2} \boldsymbol{b}\left(1-\left(1-T_{A B}(\boldsymbol{b}) \sigma_{i n}^{N N}\right)^{A B}\right) \approx \int \mathrm{d}^{2} \boldsymbol{b}\left(1-\exp \left(-A B T_{A B}(\boldsymbol{b}) \sigma_{i n}^{N N}\right)\right) .
$$

In ref. [21] this is called optical limit for the inelastic cross section.

The geometrical factor in eq. (15) which multiplies the $p+p$ differential cross section to yield the photon multiplicity equals $24.3 / \mathrm{mb}$ for the $10 \%$ most central $\mathrm{Pb}+\mathrm{Pb}$ collisions, as analysed by WA98 (corresponding to $b_{m} \approx 4.8 \mathrm{fm}$ in our calculation). For minimum bias $p+\mathrm{C}$ it is $0.056 / \mathrm{mb}$, and $0.127 / \mathrm{mb}$ for $p+\mathrm{Pb}$. These values allow to calculate the yield of prompt photons in $p+A$ collisions at SPS energy from the cross section in $p+p$ as shown in Fig. 囵.

For nominal BNL-RHIC energy, $\sqrt{s}=200 A \mathrm{GeV}$, using $\sigma_{i n}^{N N}=40 \mathrm{mb}$, we obtain for 
that factor $0.119 / \mathrm{mb}$ for $p+\mathrm{Au}, 0.048 / \mathrm{mb}$ for $p+\mathrm{C}, 0.062 / \mathrm{mb}$ for $p+\mathrm{S}$, and finally $23.1 / \mathrm{mb}$ for $\mathrm{Au}+\mathrm{Au}$ (with $10 \%$ centrality cut; $\sigma_{\text {tot }}^{\mathrm{AuAu}}=7024 \mathrm{mb} ; b_{m} \approx 4.7 \mathrm{fm}$ ).

\section{NUCLEAR BROADENING OF THE INTRINSIC $K_{T}$ DISTRIBUTION}

The nuclear broadening of the intrinsic transverse momentum distributions of the partons was observed experimentally by the broadening of the transverse momentum distribution of final state particles. For example, ref. [23] analyzed $p_{t}$ distributions of mesons made of light quarks $(\pi, \mathrm{K}, \ldots)$; ref. 《4] analyzed Drell-Yan pairs and quarkonium states $(J / \psi$ and $\Upsilon)$.

The aim of this paper is to discuss whether that broadening can explain the discrepancy of the WA98 data for $\mathrm{Pb}+\mathrm{Pb}$ collisions to simple extrapolations of proton-proton data and calculations. We define the broadening as

$$
\Delta k_{t}^{2} \equiv\left\langle k_{t}^{2}\right\rangle_{A B}-\left\langle k_{t}^{2}\right\rangle_{p p},
$$

where $\left\langle k_{t}^{2}\right\rangle_{A B(p p)}$ is the average transverse momentum in $A+B(p+p)$ collisions. For $\mathrm{Pb}+\mathrm{Pb}$ collisions values of $\Delta k_{t}^{2}=0-1 \mathrm{GeV}^{2}$ will be used in our calculations below. Larger broadening, as suggested for example by ref. [24] for hot QCD matter, seem to be ruled out at SPS energies as will be shown in section $\square$. The value suggested in [24] for cold (hot) QCD matter is

$$
\Delta k_{t}^{2}=0.2(3.0) \mathrm{GeV}^{2} \cdot \frac{L}{10 \mathrm{fm}}
$$

$L$ is the length of the QCD medium through which the parton propagates before the hard process. The average thickness of a sphere is the volume divided by the area:

$$
\langle L\rangle_{\text {sphere }}=\left(\frac{4}{3} \pi R^{3}\right) /\left(\pi R^{2}\right)=\frac{4}{3} R_{A, B} .
$$

For proton-nucleus collisions that value has to be divided by two, since we are interested in initial state interaction only (the outgoing photon does not interact strongly). For nucleus-

nucleus collisions another factor two is necessary, since both the intrinsic transverse momentum of the projectile and of the target can increase due to soft inital-state interac- 
tions. $R_{A, B}$ denotes the radius of the projectile (target) nucleus. For $\mathrm{Pb}+\mathrm{Pb}$ this means $L \simeq 2 \cdot \frac{2}{3} R=\frac{4}{3} \cdot 6.6 \mathrm{fm}=8.8 \mathrm{fm}$. From eq. (18) we thus obtain for cold (hot) QCD matter

$$
\Delta k_{t}^{2}=0.2(3.0) \mathrm{GeV}^{2} \cdot 0.88=0.176(2.64) \mathrm{GeV}^{2} .
$$

For gluons $\Delta k_{t}^{2}$ should be larger by the ratio of the Casimir operators of the octet and triplet representations of color-SU(3), $=9 / 4$. While these number are only rough estimates of the order of magnitude, we shall see below that $\Delta k_{t}^{2} \sim 0.5-1 \mathrm{GeV}^{2}$ is in the range needed to explain the WA98 prompt photon excess for $p_{t} \gtrsim 2.5 \mathrm{GeV}$.

Such small broadening is also compatible with the fits to $p+A$ data at Fermilab energies 沺. Those fits yield

$$
\Delta k_{t}^{2}=0.133(0.027) \mathrm{GeV}^{2}\left(\left(\frac{A}{2}\right)^{\frac{1}{3}}-1\right)
$$

for $J / \psi$ (Drell-Yan pair) production. For $p+\mathrm{Pb}$ this is $\Delta k_{t}^{2}=0.49(0.10) \mathrm{GeV}^{2}$. Assuming linear scaling [24] of $\Delta k_{t}^{2}$ with the length traversed through the QCD matter, $L$, the above value has to be multiplied by two, for minimum bias lead on lead collisions:

$$
\Delta k_{t}^{2}(\mathrm{~Pb}+\mathrm{Pb})=0.98(0.2) \mathrm{GeV}^{2} .
$$

Regarding the Drell-Yan process, the $\Delta k_{t}^{2}$ broadening of the quarks in the initial state equals that of the muon pair in the final state. In our case, however, the dominant processes involve a gluon in the initial state, and so the broadening in the initial state is expected to be larger by a factor $(1+9 / 4) /(1+1)$, i.e. $0.2 \mathrm{GeV}^{2} \rightarrow 0.325 \mathrm{GeV}^{2}$. For a central trigger, $L$ and thus $\Delta k_{t}^{2}$ could even be somewhat larger.

To summarize, values for $\Delta k_{t}^{2}$ up to $1 \mathrm{GeV}^{2}$ appear to be within the range given by various fits to data, and shall be employed below for the computation of prompt photon production in nuclear collisions. 


\section{RESULTS FOR CERN-SPS ENERGY}

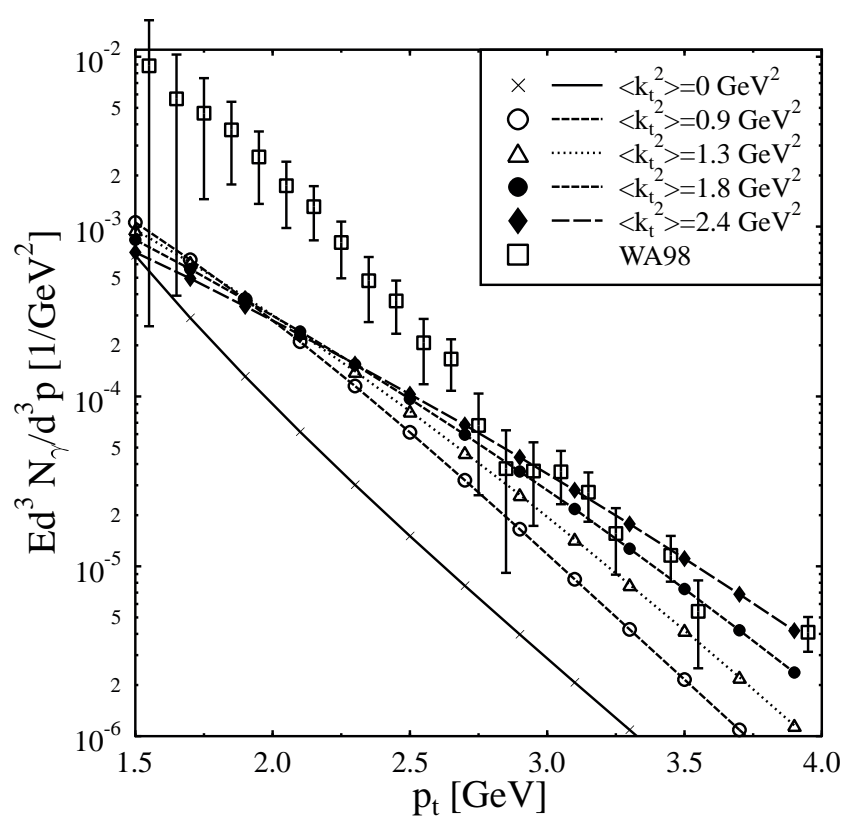

FIG. 2. The transverse momentum distribution of prompt photons at center-of-mass rapidity in the $10 \%$ most central $\mathrm{Pb}+\mathrm{Pb}$ reactions at $\sqrt{s}=17.4 A \mathrm{GeV}$. The data [1] is compared to calculations for various intrinsic $\left\langle k_{t}^{2}\right\rangle$.

Fig. 2 depicts the differential multiplicity of direct photons vs. transverse momentum in central $\mathrm{Pb}+\mathrm{Pb}$ reactions at $\sqrt{s}=17.4 A \mathrm{GeV}$. We compare calculations with various values for the intrinsic transverse momentum, $\left\langle k_{t}^{2}\right\rangle$, all corresponding to $K=2$. The data are from ref. [1]. Again, as was the case for $p+p$ collisions at similar energy, the calculation assuming pure DGLAP evolution underestimates the data strongly. However, the "standard" intrinsic transverse parton momentum of $\left\langle k_{t}^{2}\right\rangle \simeq 1.3 \mathrm{GeV}^{2}$ extracted already on the $p+p$ level (see section [I] improves the agreement a lot. Within the experimental error bars, the WA98 data leaves room for up to $\Delta k_{t}^{2} \sim 1 \mathrm{GeV}^{2}$ additional broadening from nuclear effects. However, we can also conclude that transverse momentum broadening much beyond $\Delta k_{t}^{2} \simeq 1 \mathrm{GeV}^{2}$ is not seen in the WA98 data.

Another interesting result is that the calculated photon spectrum below $p_{t} \lesssim 2.5 \mathrm{GeV}$ 
depends only weakly on the amount of intrinsic transverse momentum. That is because of the cut-off on the momentum transfer, eq. (4). Therefore, the excess photons seen in the data, as compared to our calculation, can be attributed to small-momentum transfer scatterings (final-state interactions). The computation of that contribution to the photon yield is, however, out of the scope of the present manuscript; see e.g. [25] for recent work, and references therein.

\section{RESULTS FOR BNL-RHIC ENERGY}

We now turn to nuclear collisions at collider energy. Here, we focus on $\mathrm{Au}+\mathrm{Au}$ collisions at $\sqrt{s}=200 A \mathrm{GeV}$ and central rapidity, $y \approx 0$. (Estimates for $p+A$ collisions can be obtained from our $\mathrm{Au}+\mathrm{Au}$ results by an appropriate scaling as explained in section III.) At relatively small transverse momenta nuclear shadowing effects [11] may reduce the prompt photon cross section somewhat [12,13]. However, for $p_{t} \gtrsim 2 \mathrm{GeV}$ the effect is not very large, $\lesssim 20 \%$, and we shall not investigate it in more detail here. On the other hand, it has been suggested that at high energies the gluon distribution in nuclei may saturate at small fractional light-cone momentum $x$ [26] (but much larger $x$ than for protons). This must happen at least when the unitarity bound for the inelastic scattering of a QCD dipole off a nucleus is reached in the $x-Q^{2}$ plane [15], perhaps earlier. Simply speaking, the nucleus becomes a "black disc" in the infinite momentum frame. If heavy nuclei but not protons become black, or almost black, jets with $p_{t}<Q_{\text {black }}$ will be absorbed in the final state in $A+A$ scattering円, but not in $p+p$ scattering. So high $p_{t}$ jets will be expected for $p_{t}>Q_{\text {black }}$ only. Thus, qualitatively, the $p_{t}$ distribution should be rather different from $p+p$ at not too large $p_{t}$. The yield of jets will be suppressed but the transverse momentum distribution will be wider.

\footnotetext{
${ }^{1}$ They might produce a "saturated plasma" [27] at central rapidity which would then also contribute to photon production [25].
} 
For $A=200$ nuclei, the unitarity limit for the inelastic $q \bar{q}$-nucleus cross section is reached at $x \sim 10^{-2}$ for $Q \sim 1.5 \mathrm{GeV}$, and at $x \sim 10^{-3}$ for $Q \sim 2.5 \mathrm{GeV}$ (if nuclear shadowing is weak) [15]. If shadowing is also taken into account, the $x$-values where the unitarity limit is approached become even smaller [15]. Therefore, at rapidity $y \sim 0$ and at RHIC energy, $\sqrt{s}=200 A \mathrm{GeV}$, we expect only small effects on prompt photon production.

At high energy, the bremsstrahlung contribution to the prompt photon yield is substantial [12,13, 16]. In this process, a (anti-)quark is produced first in the central rapidity region with transverse momentum $p_{t c}$ (see section 【1). That quark then radiates an almost collinear photon with transverse momentum $p_{t}=z p_{t c}$. In principle, the quark could in fact suffer further collisions which in turn may suppress the contribution from soft (i.e., small $z$ ) bremsstrahlung to prompt photon production [13,28]. This is due to the well known LandauPomeranchuck-Migdal effect. The quark could also suffer some energy loss prior to emitting the photon. The radiative energy loss of the quark is mainly due to radiation of soft gluons. Therefore, the formation time (or the formation length) of the radiated gluons cannot be much smaller than that of the photons. Thus, quark energy loss might not interfere with the radition of the photons in the final state. In any case, the complete extinction of all bremsstrahlung would represent an upper limit for the sum of both effects [28]. This would change our result by less than a factor of two. Therefore, we do not discuss these effects in more detail here (see [13]), since their largest possible impact on the photon production cross section is smaller than that from nuclear broadening of the intrinsic transverse momentum. 


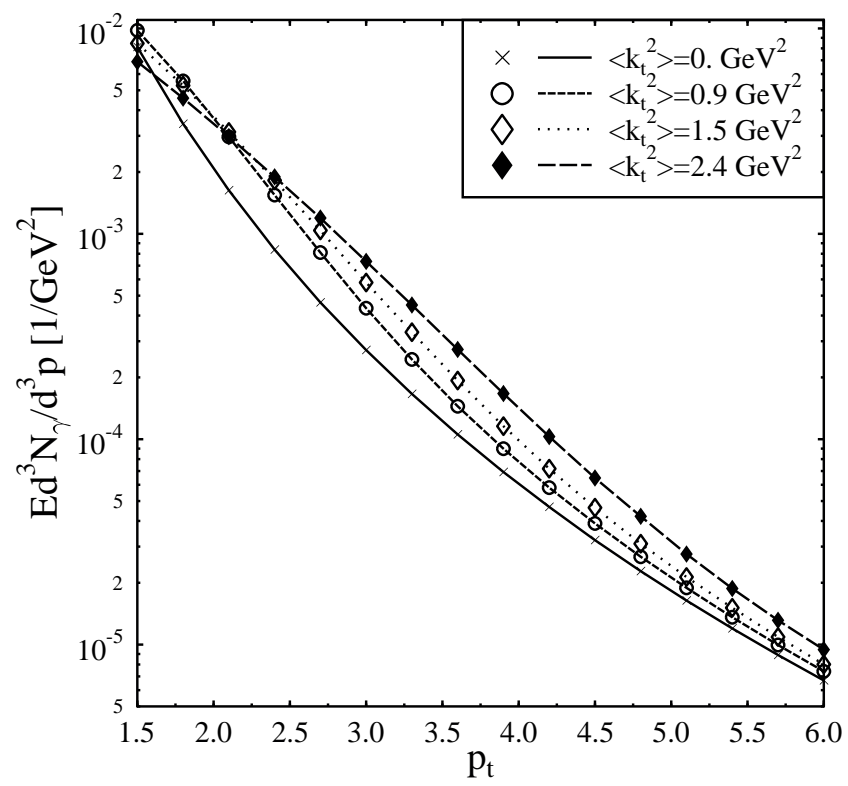

FIG. 3. The transverse momentum distribution of prompt photons at center-of-mass rapidity in the $10 \%$ most central $\mathrm{Au}+\mathrm{Au}$ reactions at $\sqrt{s}=200 \mathrm{~A} \mathrm{GeV}$ for various intrinsic $\left\langle k_{t}^{2}\right\rangle$.

In Fig. 3 we show prompt photon spectra at RHIC energy, for the $10 \%$ most central $\mathrm{Au}+\mathrm{Au}$ events at $\sqrt{s}=200 \mathrm{AGeV}$. As before, we assumed $K=2$. One observes that the effect of intrinsic transverse momentum in the initial state is much less prominent than at lower energy, in agreement with the results of [7] for pion production. The reason for this behavior is that the photon spectrum becomes "harder" at higher energy, i.e. it decreases less steeply with $p_{t}$. Nevertheless, at $p_{t} \sim 3-4 \mathrm{GeV}$ the intrinsic parton transverse momentum can increase the prompt photon multiplicity by up to a factor of 3 . That would be the most promising kinematical domain for an experimental study of the nuclear broadening of the intrinsic transverse momentum distributions. The region of smaller $p_{t}$ might be dominated by soft contributions, as indicated by the flattening of the spectra in Fig. 3. On the other hand, at larger $p_{t}$ intrinsic transverse momentum becomes less effective. 


\section{SUMMARY AND CONCLUSION}

Prompt photon data from $p+p$ scattering at $\sqrt{s}=19.4 \mathrm{GeV}$ obtained by the E704 collaboration [17] reveals that standard DGLAP evolution without intrinsic transverse momentum, and for our choice for the factorization scale $Q=2 p_{t}$, underestimates that data. Including an intrinsic transverse momentum of the initial-state partons of $\sim 1.3 \mathrm{GeV}^{2}$ allows for a fair description of the E704 data.

Further, we presented results for prompt photon $p_{t}$ distributions in nuclear collisions, assuming $A$-scaling as suggested by the geometry of the Glauber incoherent approach. At SPS energy, a good description of the data for $p_{t} \gtrsim 2.7 \mathrm{GeV}$ requires additional intrinsic $k_{t}$ of the partons in the initial state, i.e. nuclear broadening of the transverse parton distributions by $\Delta k_{t}^{2} \sim 0.5-1 \mathrm{GeV}^{2}$ (for the same factorization scale as above). Thus, the WA98 data provide evidence for the nuclear broadening effect in the $p_{t}$ distribution of prompt photons. Photons appear more appropriate than hadrons to test nuclear broadening of the intrinsic transverse momentum because they do not suffer from rescattering in the final state.

Below $p_{t} \sim 2.7 \mathrm{GeV}$, both the yield and the slope of the WA98 photon data is not described as being due to hard scatterings with momentum transfer $\geq 1 \mathrm{GeV}$. At $p_{t} \sim 2 \mathrm{GeV}$, prompt photons from hard collisions underestimate the WA98 data by almost an order of magnitude, for any amount of intrinsic $\left\langle k_{t}^{2}\right\rangle$. Thus, that kinematic domain is dominated by small momentum transfer processes.

At RHIC energy, the effect of the intrinsic transverse momentum is much less prominent. Only at $p_{t} \sim 3-4 \mathrm{GeV}$ can intrinsic parton transverse momentum increase the prompt photon multiplicity by up to a factor of 3 . That would thus be the most promising kinematical domain for an experimental study of nuclear broadening of the intrinsic transverse momentum at RHIC. Again, at $p_{t} \lesssim 2-3 \mathrm{GeV}$ the realistic prompt photon distribution (incl. intrinsic $k_{t}$ ) from large momentum transfer scattering flattens out, that region being most likely dominated by soft contributions. 


\section{ACKNOWLEDGMENTS}

We thank T. Peitzmann and M. Mora for discussions on the WA98 data, and Y. Dokshitzer, J. Jalilian-Marian and I. Vitev for discussions on energy loss and fragmentation time scales. A.D. acknowledges support from DOE Research Grant DE-FG-02-93ER-40764. M.S. thanks the US Department of Energy for financial support. L.G. would like to thank the

Josef Buchmann Foundation for financial support. H.S. was supported in parts by BMBF, DFG, and GSI.

[1] M. M. Aggarwal et al. [WA98 Collaboration], Phys. Rev. Lett. 85, 3595 (2000); and nuclex/0006007.

[2] W. Vogelsang and M. R. Whalley, J. Phys. G 23, A1 (1997).

[3] G. T. Bodwin, S. J. Brodsky and G. P. Lepage, Phys. Rev. Lett. 47, 1799 (1981); and Phys. Rev. D 39, 3287 (1989).

[4] P. L. McGaughey, J. M. Moss and J. C. Peng, Ann. Rev. Nucl. Part. Sci. 49, 217 (1999); and J. C. Peng, P. L. McGaughey and J. M. Moss, hep-ph/9905447.

[5] J. F. Owens, Rev. Mod. Phys. 59, 465 (1987); R.D. Field, Applications of Perturbative QCD (Addison-Wesley, Reading, MA, 1989).

[6] J. C. Collins, D. E. Soper and G. Sterman, Nucl. Phys. B 250, 199 (1985).

[7] X. N. Wang, Phys. Rev. Lett. 81, 2655 (1998); M. Gyulassy and P. Levai, Phys. Lett. B442, 1 (1998).

[8] M. M. Aggarwal et al. [WA98 Collaboration], Phys. Rev. Lett. 81, 4087 (1998); Erratum-ibid. 84, $578(2000)$.

[9] G. Papp, P. Levai and G. Fai, Phys. Rev. C 61, 021902 (2000). 
[10] R. Brock et al. [CTEQ Collaboration], Rev. Mod. Phys. 67, 157 (1995); H. L. Lai et al., Phys. Rev. D 55, 1280 (1997); H. L. Lai and W. K. Tung, Z. Phys. C74, 463 (1997).

[11] L. L. Frankfurt and M. I. Strikman, Phys. Rept. 160, 235 (1988).

[12] N. Hammon, A. Dumitru, H. Stöcker and W. Greiner: Phys. Rev. C57, 3292 (1998).

[13] J. Jalilian-Marian, K. Orginos and I. Sarcevic, hep-ph/0010230; hep-ph/0101041.

[14] K. J. Eskola, V. J. Kolhinen and C. A. Salgado, Eur. Phys. J. C9, 61 (1999).

[15] L. Frankfurt, V. Guzey and M. Strikman, J. Phys. G27, R23 (2001).

[16] P. Aurenche, A. Douiri, R. Baier, M. Fontannaz and D. Schiff, Phys. Lett. B 140, 87 (1984); Nucl. Phys. B 286, 553 (1987); P. Aurenche, R. Baier, M. Fontannaz and D. Schiff, Nucl. Phys. B 297, 661 (1988); P. Aurenche, P. Chiappetta, M. Fontannaz, J. P. Guillet and E. Pilon, Nucl. Phys. B 399, 34 (1993).

[17] D. L. Adams et al. [E704 Collaboration], Phys. Lett. B345, 569 (1995).

[18] C. Wong and H. Wang, Phys. Rev. C 58, 376 (1998).

[19] L. Frankfurt, M. Strikman and S. Liuti, Brookhaven 1990, Experiments and detectors for a relativistic heavy ion collider, p. 103-115.

[20] R. J. Glauber, Lectures in Theoretical Physics Vol. 1, Interscience, (1959) p. 315

[21] W. Czyz and L. C. Maximon, Annals Phys. 52, 59 (1969).

[22] C. W. deJager, H. deVries und C. deVries, Atomic Data and Nuclear Data Tables 14, 479 (1974).

[23] J. W. Cronin, H. J. Frisch, M. J. Shochet, J. P. Boymond, R. Mermod, P. A. Piroue and R. L. Sumner, Phys. Rev. D 11, 3105 (1975).

[24] R. Baier, Y. L. Dokshitzer, A. H. Mueller, S. Peigne and D. Schiff, Nucl. Phys. B 484, 265 (1997); R. Baier, D. Schiff and B. G. Zakharov, Ann. Rev. Nucl. Part. Sci. 50, 37 (2000). 
[25] P. Aurenche, F. Gelis, R. Kobes and H. Zaraket, Phys. Rev. D 58, 085003 (1998); M. G. Mustafa and M. H. Thoma, Phys. Rev. C 62, 014902 (2000); Erratum: hep-ph/0103293; K. Gallmeister, B. Kämpfer and O. P. Pavlenko, Phys. Rev. C 62, 057901 (2000); J. Alam, S. Sarkar, T. Hatsuda, T. K. Nayak and B. Sinha, Phys. Rev. C 63021901 (2001).

[26] L. McLerran and R. Venugopalan, Phys. Rev. D49, 2233 (1994); and ibid. D49, 3352 (1994); A. H. Mueller, Nucl. Phys. B 558, 285 (1999).

[27] K. J. Eskola, K. Kajantie, P. V. Ruuskanen and K. Tuominen, Nucl. Phys. B 570, 379 (2000); A. Dumitru and M. Gyulassy, Phys. Lett. B 494, 215 (2000); G. C. Nayak et al., Nucl. Phys. A 687, 457 (2001); J. Bjoraker and R. Venugopalan, Phys. Rev. C 63, 024609 (2001); R. Baier, A. H. Mueller, D. Schiff and D. T. Son, Phys. Lett. B 502, 51 (2001).

[28] A. Dumitru and N. Hammon, hep-ph/9807260. 